\title{
Chopping-Out-Of-band (COOB) for reducing ripple in chopper amplifiers
}

\author{
Tong Ling ${ }^{1}$, Huajun Fang ${ }^{1 a)}$, Xiao Zhao ${ }^{2}$, and Jun Xu ${ }^{1}$ \\ ${ }^{1}$ Institute of Microelectronics, Tsinghua University, Beijing 100084, P.R.China \\ ${ }^{2}$ School of Geophysics and Information Technology, \\ China University of Geosciences (Beijing), Beijing 100083, P.R.China
}

a) hjfang@tsinghua.edu.cn

\begin{abstract}
This paper introduces a method named Chopping-Out-Of-band (COOB), which can suppress ripple in chopper amplifiers. By choosing a suitable chopping frequency $\left(f_{\text {chop }}\right.$ ) located in $G B W<f_{\text {chop }}<N L B W$ (NoLoad Bandwidth), the ripple will be suppressed rather than amplified and the effect can be improve further by an extra filter. The COOB technique was employed in instrumentation amplifiers as examples and the extra filter was designed to passive filter. These chopper amplifiers were simulated on UMC 0.18 um technology with the help of Cadence SpectreRF. Simulation results showed that the ripple was suppressed by $-93.4 \mathrm{~dB}$ in Power Spectral Density (PSD) analysis without additional power consumption.
\end{abstract}

Keywords: chopper-stabilized, chopping-out-of-band, offset, 1/f noise, ripple reduction

Classification: Integrated circuits

\section{References}

[1] C. C. Enz and G. C. Temes: Proc. IEEE 84 (1996) 1584. DOI:10.1109/5.542410

[2] R. Wu, K. A. A. Makinwa and J. H. Huijsing: IEEE J. Solid-State Circuits 44 (2009) 3232. DOI:10.1109/JSSC.2009.2032710

[3] Y. Kusuda: IEEE J. Solid-State Circuits 45 (2010) 1436. DOI:10.1109/JSSC. 2010.2048142

[4] Q. Fan, J. Huijsing and K. A. A. Makinwa: ISSCC Dig. Tech. Papers (2013) 176. DOI:10.1109/ISSCC.2013.6487688

[5] I. Akita and M. Ishida: ISSCC Dig. Tech. Papers (2013) 178. DOI:10.1109/ ISSCC.2013.6487689

\section{Introduction}

In CMOS technology, offset and $1 / \mathrm{f}$ noise have become the main circuit errors at very low frequency. Dynamic chopping technique is widely used for cancelling the offset and $1 /$ f noise in high precision CMOS amplifiers. The offset and $1 / \mathrm{f}$ noise in chopper amplifiers are modulated to a higher frequency while the signal is demodulated on the baseband. However, chopping also brings imperfections such 
as ripple and gain losing [1]. It has been reported that the ripple can achieve $48 \mathrm{mV}$ at the output of the amplifier [2], undoubtedly this is unacceptable. There have been many papers which are reported in recent years show different ripple reduction techniques $[2,3,4,5]$. On the other hand, in [1], the author describes that the chopping frequency $\left(f_{\text {chop }}\right)$ is limited by finite bandwidth and delay introduced by the main amplifier. In this paper, we point out that the speed of chopping is not influenced by the load capacitances $\left(C_{L}\right)$ in the chopping nodes. As a consequence, a ripple reduction technique named COOB is proposed, which sets the $f_{\text {chop }}$ locate in the outside of GBW so that the ripple can be suppressed. Furthermore, an extra passive filter is employed to enhance the suppression. The COOB technique would not introduce extra power consumption and open-loop gain losing.

\section{Proposed COOB}

The operational transconductance amplifier (OTA), which is used as the first stage in instrumentation amplifiers, provides the main offset and $1 / \mathrm{f}$ noise. As a consequence the chopping is usually implemented on OTA, which is shown in Fig. 1. The input signal (a) will be modulated to (b) and then amplified (c). The speed of chopping is limited to that whether the signal (b) could be amplified smoothly. It is worth noting that some load capacitors (Fig. 1) or compensation capacitors (Fig. 4) appeared at the chopping nodes so that we must take them into account.

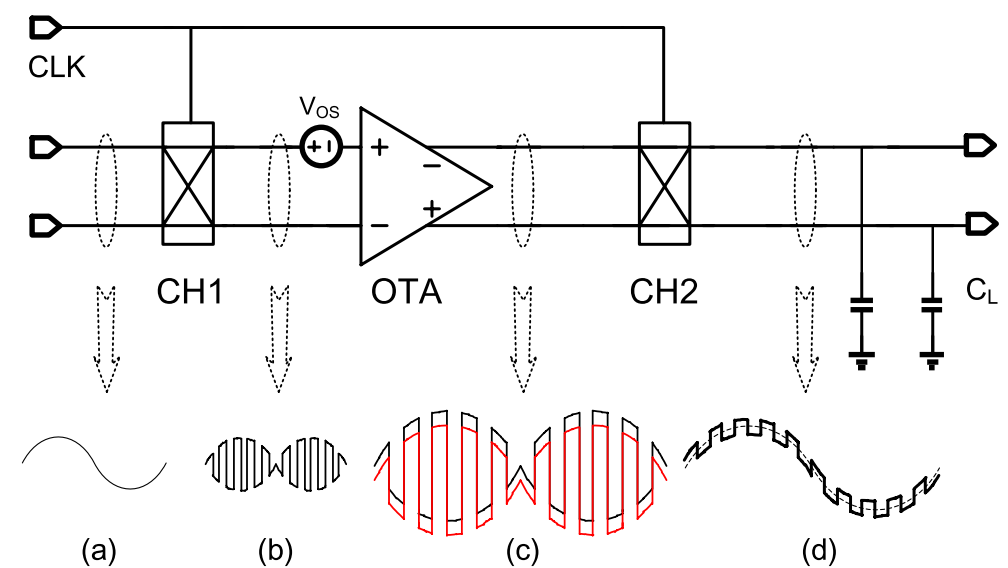

Fig. 1. The chopping theory.

As Fig. 2(a) describes, the OTA is amplifying a DC signal. The red line means high voltage while the blue means low voltage. At the moment when clock changes, shown in Fig. 2(b) and (c), the $C_{L}$ remains unchanged while the OTA changes to its opposite state. That means the speed of chopping is not limited by the coaction of $C_{L}$ and OTA but limited by the OTA itself. One step further, the $f_{\text {chop }}$ should be set up to lower than the no-load bandwidth (NLBW) in order to get a maximum gain. The no-load ac response of OTA is described in Fig. 3.

With the help of modulators, offset will be modulated only once and then appears at the output (Fig. 1), this is the ripple. It is obviously that ripple will be suppressed by $C_{L}$. The ac response with $C_{L}$ and without $C_{L}$ are given in Fig. 3(b). The $f_{\text {chop }}$ is set up to: 

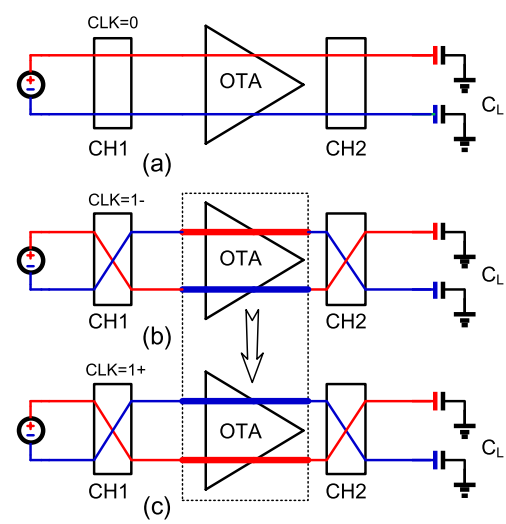

Fig. 2. Process of clock changing in the chopper amplifier.

$$
G B W<f_{\text {chop }}<N L B W .
$$

The ripple is suppressed rather than amplified. Meanwhile, this choice of $f_{\text {chop }}$ makes that a maximum gain is obtained. In general, the $C_{L}$ are compensation capacitors such as $C_{C 1}$ in Fig. 4, by which the GBW of the OTA is determined. In order to get a more effective ripple reduction, a low pass filter (LPF) is employed which also located on the outside of GBW, shown in Fig. 4. The enhanced suppression is described in Fig. 3(c).
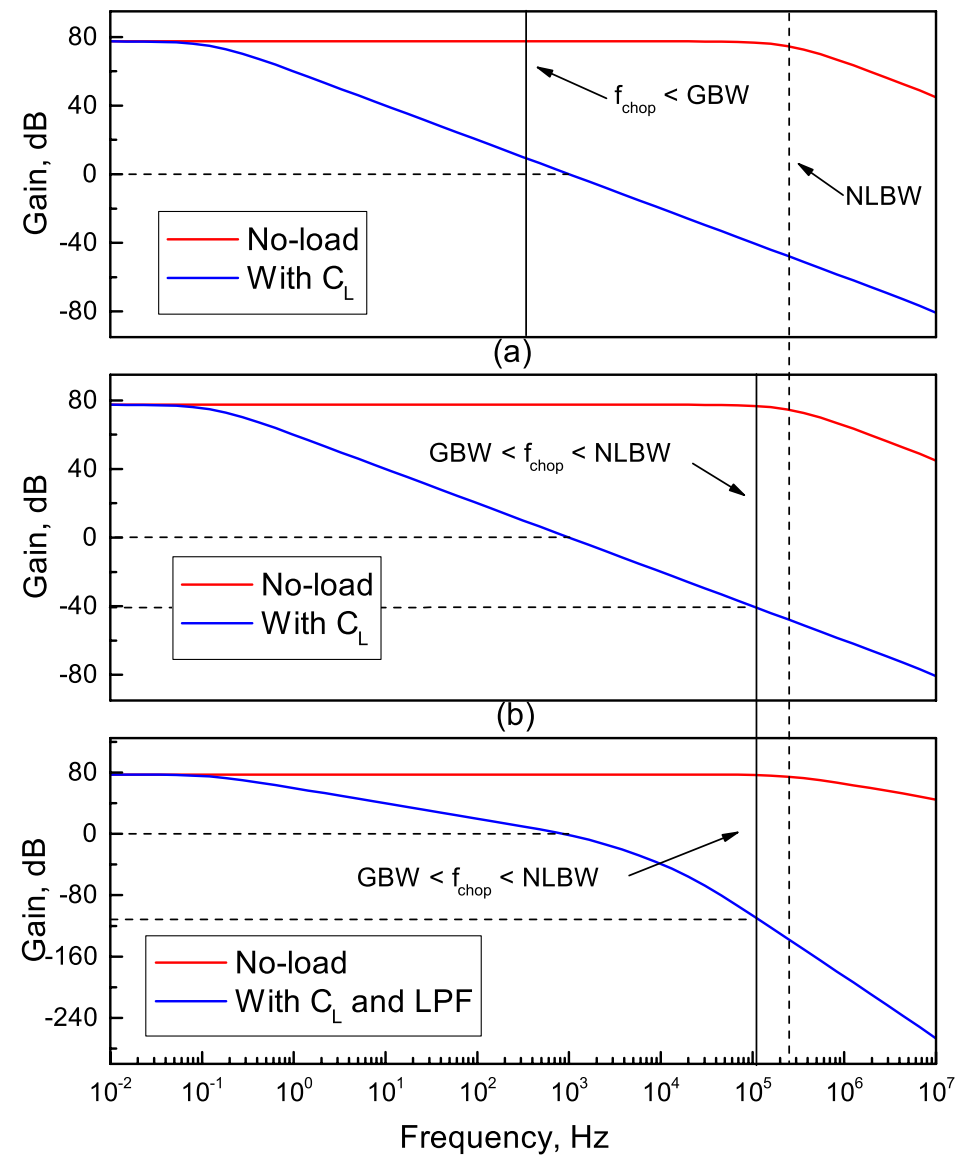

(c)

Fig. 3. AC response of OTA, a) Conventional chopping, b) Proposed COOB, c) Proposed COOB with LPF. 


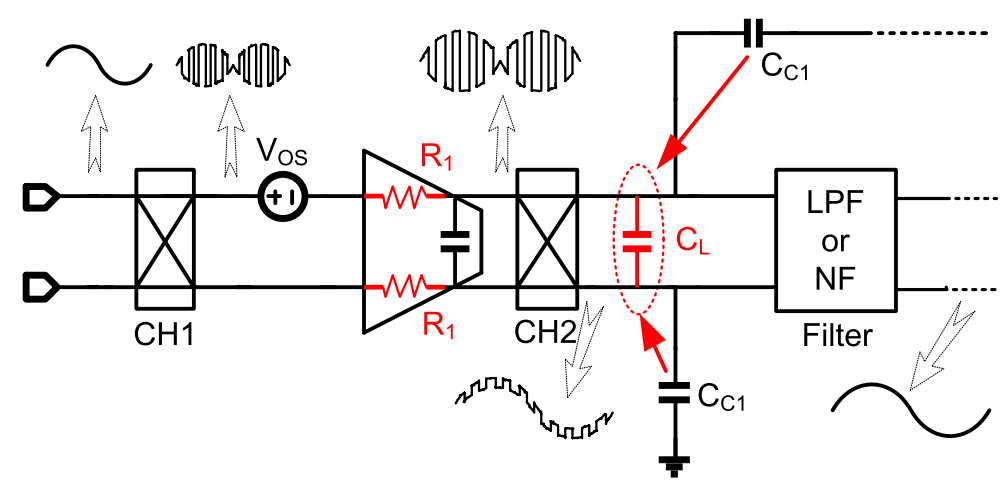

Fig. 4. COOB structure with filter.

Assuming that $P_{0}$ is the dominant pole which determined by OTA and $C_{C 1}, P_{1}$, $P_{2}, P_{3}$ are introduced by the LPF, and $A_{v 1}$ is the DC gain of the OTA. Ignoring the interaction of poles, the ripple suppression could be described by $\frac{V_{\text {ripple }}}{V_{\text {offset }} \text { : }}$

$$
\frac{V_{\text {ripple }}}{V_{\text {offset }}}=-80 \lg \left(f_{\text {chop }}\right)+20 \lg \left(P_{0} \cdot P_{1} \cdot P_{2} \cdot P_{3}\right)+A_{v 1}-3 .
$$

With the help of LPF, the ripple will get an extra $-60 \mathrm{~dB} / \mathrm{dec}$ roll-off reduction. A notch filter (NF) located on the outside of GBW could also meet the needs as well as LPF, which can select some specific frequency out.

\section{Instrumentation amplifier with $\mathrm{COOB}$}

The proposed COOB has been employed in instrumentation amplifiers shown in Fig. 5. These amplifiers consist of 2 signal paths, a low frequency path and a high frequency path. The low frequency path provides the main DC gain, and chopping is used for cancelling the offset and $1 / \mathrm{f}$ noise with COOB to reduce the ripple. The high frequency path makes the overall amplifier stable and generates a suitable
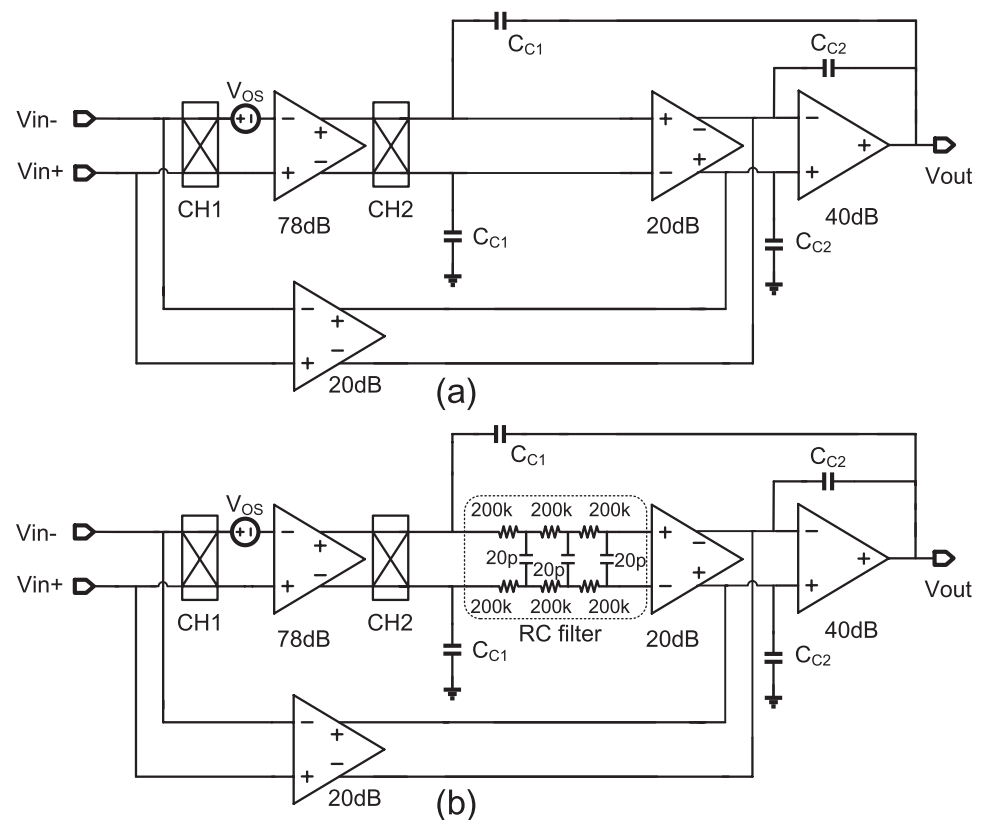

Fig. 5. Instrumentation amplifiers with chopping, a) Conventional instrumentation amplifier, b) COOB instrumentation amplifier with LPF. 
phase margin [4]. The GBW of the chopping stage $\left(G B W_{1}\right)$ and GBW of the overall circuit are determined by $C_{C 1}$ and $C_{C 2}$, respectively. The ripple will be suppressed by COOB structure and then amplified by $A_{v 2}$ and $A_{v 3}$. However, $A_{v 2} \cdot A_{v 3}$ is smaller than $\frac{V_{\text {ripple }}}{V_{\text {offiset }}}$ so that the ripple in COOB instrumentation amplifier is still been suppressed.

The LPF is designed to a RC filter which provides $P_{1}, P_{2}$ and $P_{3}$. Because the dominant pole $P_{0}$ is determined by OTA and $C_{C 1}$, the parameters of the RC filter are acceptable in IC. On the other hand, because of the high frequency path, the stability of the overall circuits will not be influenced by the filter, so that the precision and matching of the RC filter are not concerned. Moreover, the RC filer consumes no additional power.

\section{Simulation results}

Two instrumentation amplifiers AMP1 and AMP2 were designed shown in Fig. 5(a) and (b), and the amplifiers were simulated on UMC 0.18 um CMOS process with the help of spetreRF. In order to observe the ripple reduction, these amplifiers were used as $20 \mathrm{~dB}$ gain closed-loop amplifiers, and the $V_{O S}$ was set up to $10 \mathrm{mV}$. After the transient analysis, the output signal were calculated by Power Spectral Density (PSD) function. The transient output signal and their PSD are shown in Fig. 6. We have obtained 3 groups of data: AMP1 with $f_{\text {chop }}=1 k\left(<G B W_{1}\right)$, AMP1 with $f_{\text {chop }}=200 k\left(>G B W_{1}\right)$, AMP2 with $f_{\text {chop }}=200 k\left(>G B W_{1}\right)$.

According to the transient simulation results, the ripple has been cancelled obviously, from peak-to-peak voltage $216 m V_{p-p}$ (In Band), $114.4 m V_{p-p}$ (Out Of Band), to $18.2 \mu V_{p-p}$ (Out Of Band with LPF). Compared with chopping in band, the PSD results showed that the ripple has been reduced by $-17.5 \mathrm{~dB}$ and
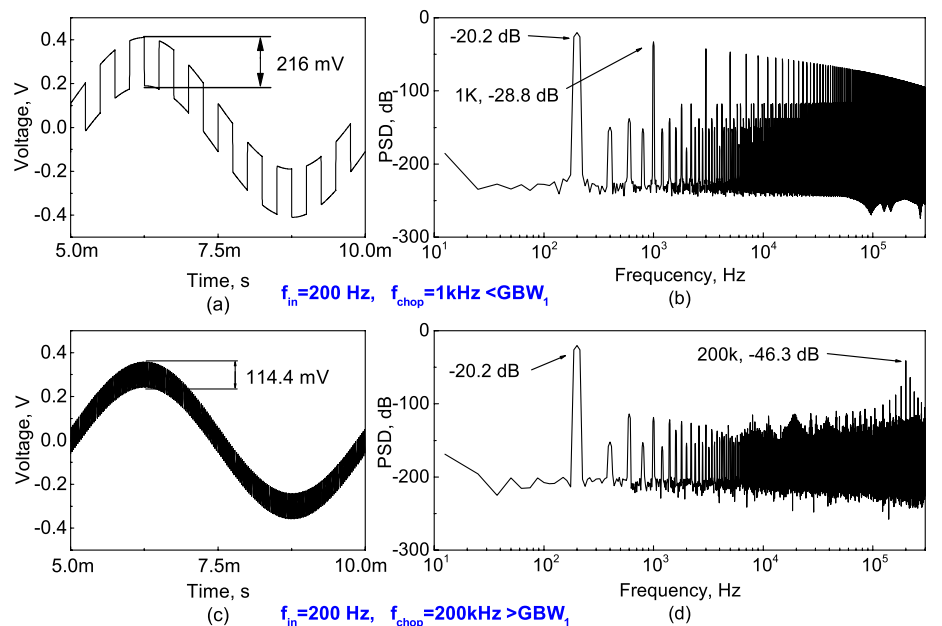

(c) $f_{\text {in }}=200 \mathrm{~Hz}, f_{\text {chop }}=200 \mathrm{kHz}>\mathrm{GBW}$

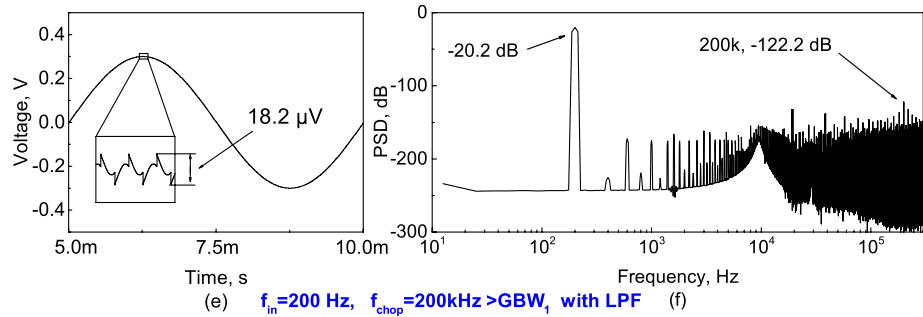

Fig. 6. Ripple in transient simulation and its PSD results, $V_{O S}=$ $10 \mathrm{mV}$. 
$-93.4 \mathrm{~dB}$. The simulation results of key parameters of the two amplifiers are listed in Table I.

Table I. Performance summary of AMP1 and AMP2

\begin{tabular}{|c|c|c|c|}
\hline Parameter & \multicolumn{2}{|c|}{$A M P 1$} & $A M P 2$ \\
\hline Voltage supply (V) & \multicolumn{2}{|l|}{1.8} & 1.8 \\
\hline$C_{L}(\mathrm{pF})$ & \multicolumn{2}{|l|}{20} & 20 \\
\hline Power $(\mu \mathrm{A})$ & \multicolumn{2}{|l|}{148} & 148 \\
\hline DC Gain (dB) & \multicolumn{2}{|c|}{131.4} & 131.4 \\
\hline$G B W_{1}(\mathrm{kHz})$ & \multicolumn{2}{|c|}{1.19} & 1.19 \\
\hline Overall GBW (kHz) & \multicolumn{2}{|c|}{1200} & 1210 \\
\hline PM (deg) & \multicolumn{2}{|l|}{73} & 73 \\
\hline Chopping Mode & Chopping In Band & COOB & COOB \\
\hline$f_{\text {chop }}(\mathrm{kHz})$ & 1 & 200 & 200 \\
\hline Gain with chopping (dB) & 130.9 & 128.5 & 128.5 \\
\hline$V_{\text {ripple }}$ & $216 m V_{p-p}$ & $114.4 m V_{p-p}$ & $18.2 \mathbf{u} \mathbf{V}_{p-p}$ \\
\hline
\end{tabular}

\section{Conclusion}

A method for cancelling the ripple in chopper amplifiers named COOB is proposed in this paper. With the COOB technique, two instrumentation amplifiers were designed as examples. Simulation results showed that the ripple can be reduced by $-93.4 \mathrm{~dB}$, from $216 m V_{p-p}$ to $18.2 \mu V_{p-p}$ without additional power consumption.

\section{Acknowledgments}

This work is supported by the NSFC (grant No. 11227202). 